\title{
Tip/Tilt point spread function reconstruction for laser guide star multi conjugate adaptive optics
}

\author{
L. Gilles ${ }^{1}$, C. Correia ${ }^{2}$, J.P. Véran ${ }^{2}$, L. Wang ${ }^{1}$ and B.L. Ellerbroek ${ }^{1}$ \\ ${ }^{1}$ Thirty Meter Telescope Observatory Corp., 1200 E. California Blvd, MC 102-8, \\ Pasadena, CA 91125, USA \\ ${ }^{2}$ NRC, Herzberg Institute of Astrophysics, 5171 W. Saanich Road, Victoria, BC V9E 2E7, Canada
}

\begin{abstract}
In adaptive optics systems employing laser guide stars, the tip/tilt contribution to the long exposure point spread function must be estimDed separately from the hiJh-order tip/tilt removed point spread function because this component is estimated separately from a single or multiple low-order natural guide star wavefront sensors. This paper investigates this problem for laser guide star multi conjugate adaptive optics. The approach is bDed on the scheme developed by Flicker in 2003 [1], and consists in post-processing the measurement covariance matrix of multiple low-order natural guide star wavefront sensors controlling tip/tilt and tilt anisoplanatism. An innovative simulation model based "balanced" algorithm is introduce d to capture error teนns not acc ounted for in Flicker's algorithm. Sample enclosed energy results for the Thirty Meter Telescope multi conjugate adaptive optics system demonstrate the superiority of the balanced method and call for further analytical work and experimental validation.
\end{abstract}

Keywords: Adaptive optics, laser guide star, multi conjugate, point spread function reconstruction, atmospheric turbulence

\section{INTRODUCTION}

Point spread function (PSF) knowledge is critical for any existing or proposed adaptive optics (AO) astronomical science program aiming at obtaining high angular resolution information. Examples of such programs include photometry and a strometry in crowded and sparse stellar fields, detection and characterization of exoplanets, determination of precision orbits at the Galactic Center to test general relativity and black hole growth models, dynamics of early galaxies, and gravitational lensing $[2,3,4,5,6]$. In order to enable such science programs, AO systems on existing telescopes and future extremely large telescopes are required to meet tight photometry and ast rometry budgets. For instance, the multi conjugate adaptive optics (MCAO) system under design for the Thirty Meter Telescope (TMT) is required to provide $2 \%$ differential photometry over a 30" field of view (FoV) for a 10 min integration at a wavelength of 1 micron, and 50 microarcsec root-mean-square (RMS) time dependent differential astrometry over the same FoV for a $100 \mathrm{sec}$ integration in $\mathrm{H}$ band [7,8].

Véran pioneered AO PSF reconstruction (PSFR) in 1997 by developing a practical mHhod to reconstruct the long exposure PSF of a bright natural guice star (NGS) from the accumulated wavefront sensor (WFS) $\mathrm{m}$ easurement covariance matrix [9]. Fusco in 2000 [9], and later Britton in 2005 [11], extended PSFR to capture a key missing component: angular anisoplanatism, providing the astronomical AO community a complete PSFR tool for classical NGS single-conjugate AO (SCAO) observations. 4\% Strehl ratio (SR) errors in K-band are reported on the Palomar AO system (order $16 \times 16$ ) at the $5 \mathrm{~m}$ Hale Telescope for observations of a bright star separated by 21" from its companient star. Measurements of the turbulence profile provided the necessaU input for the angular anisoplanatism filter computation in these expe riments. Recently, Jolissaint repo rted 5\% K-band SR error at the $10 \mathrm{~m}$ Keck Telescope for bright NGS AO observations [12] and stressed two critical steps to achieve this level of performance: estimation of the static/quasi-static optical aberrations (common and non-common path), and knowledge of the atmospheric turbulence profile. Flicker, in 2008, pushed the theory further to include focal anisoplanatism (i.e. the cone effect) and tip/tilt (TT), opening the door to PSFR for laser guide star (LGS) SCAO [13,1].

In LGS MCAO, multiple low-order NGS wavefront sensors (WFSs) are required in order to sense and control a few low-order atmospheric null-modes unsensed by the multiple high-order LGS WFSs. These null-modes consist of global TT and tilt anisoplanatism (TA) modes producing absolute and differential magnification on the science focal plane [14]. This paper discusses the problem of reconstructing the TT covariance matrix of these modes in the science direction of 
interest, and therefore the TT contribution to the long exposure PSF, from the multi-NGS WFS measurement covariance matrix. The approach is based on the scheme developed by Flicker in 2003 [1]. An innovative simulation model based balanced algorithm is introduced to capture error terms (tilt anisoplanatism between the NGS and science directions and spatial aliasing from uncorrected high-order wavefront errors outside the LGS metapupil) not accounted for in Flicker's algorithm. Sample enclosed energy results for the TMT LGS MCAO system demonstrating the superiority of the balanced method and calling for further analytical and experimental work are discussed.

\section{TIP/TILT PSFR FOR LGS MCAO}

The reconstructed atmospheric AO system optical transfer function (OTF, i.e. Fourier transformed PSF) is expressed at each science field point of interest as follows:

$$
\widehat{O T F}^{s y s}=\widehat{K}_{T T}^{s y s} \widehat{O T F}_{T T R}^{s y s},
$$

where $\widehat{O T F}_{\text {TTR }}^{\text {sys }}$ denotes the tip/tilt removed (TTR) OTF estimated from high-order telemetry data [15] (not discussed in this paper), and $\hat{K}_{T T}^{s y s}$ the TT blurring filter function, expressed in terms of the TT structure function (SF) as follows:

$$
\widehat{K}_{T T}^{s y s}(u)=e^{-\hat{D}_{I T}^{\wedge s}(\lambda u) / 2}, \hat{D}_{T T}^{s y s}(\lambda u)=16(\lambda / D)^{2} u^{T} \hat{C}_{T T}^{s y s} u .
$$

In these expressions, hats denote reconstructed quantities, $\lambda$ denotes the wavele ngth, $D$ the telescope di ameter and $u=\left(u_{x}, u_{y}\right)$ the angular frequency variable. $\hat{C}_{T T}^{s y s}$ is the reconstructed modal TT system phase covariance matrix (2x2, in units of radians squared). The total OTF is the product of (2.1) with a tip/tilt removed (TTR) OTF estimated the highorder LGS WFS.

We define an "unbalanced" reconstruction formula for the modal TT system phase covariance matrix appearing in (2.1), following the scheme developed by Flicker [1], which involves the following 3 steps: (i) noise removal from the global low-order m ulti-NGS measurement covariance matrix, (ii) tom ographic null-modes reconstruction (typi cally $N_{m N G S}=5$ modes are reconstructed, consistinJ of TT and 3 quadratic modes defined on two layers), and (iii) reconstructed null-modes projection onto TT along the science direction of interest. These 3 operations can be expressed as follows:

$$
\hat{C}_{T T}^{u, s y s}=H_{T T} R_{N G S}^{s y s}\left(C_{g N G S}^{s y s}-\hat{C}_{g N G S n s e}^{s y s}\right)\left(R_{N G S}^{s y s}\right)^{T}\left(H_{T T}\right)^{T},
$$

where $R_{N G S}^{s y s}$ denotes the $N_{m N G S} \times N_{g N G S}$ modal tomographic NGS phase reconstruction matrix $\left(N_{g N G S}=12\right.$ for a sy stem employing 2 TT Shack-Hartmann WFSs and 1 TT/focus/astigmatism (TTFA) i.e. an order $2 \times 2$ Shack-Hartmann WFS), $H_{T T}$ is a $2 \times N_{m N G S}$ matrix that proj ects the reconstructed null-modes onto TT along the science direction of interest, $C_{g N G S}^{s y s}$ is the closed loop NGS WFS measurement matrix, and $\hat{C}_{g N G S n s e}^{s y s}$ denotes the estIP ated measurement noise covariance matrix, computed from centroid weights and suba perture time averaged pixels intensities [16,17]. Note that the noise removal step mDy not always produce a symmetric positive definite (SPD) covariance mDrix, hence an eigenvalue decomposition is required to null possible negative eigenvalues.

We now introduce an innovat ive simulation model based "balanced" TT reconstruction formula to account for tip/tilt anisoplanatism and spatial aliasing from uncorrected high-order wavefront errors outside the LGS metapupil not captured in (2.2). SiP ulation model refers here to a high fidelity end-to-end Monte Carlo simulation of the complete LGS MCAO system. The formula reads:

$$
\widehat{K}_{T T}^{b, s y s}=\left(\widehat{K}_{T T}^{u, s y s} / \widehat{K}_{T T}^{u, \bmod }\right) K_{T T}^{\bmod },
$$

where all TT filters are expressed as in (2.1) with : 


$$
\hat{C}_{T T}^{b, s y s}=\hat{C}_{T T}^{u, s y s}+C_{T T}^{\text {mod }}-\hat{C}_{T T}^{u, \text { mod }},
$$

where $C_{T T}^{\text {mod }}$ denotes the simulation model TT covariance matrix in the science direction of interest, and $\hat{C}_{T T}^{u \text {,mod }}$ the estimate computed from the simulation model NGS WFS telemetry data as in (2.2):

$$
\hat{C}_{T T}^{u \text { mod }}=H_{T T} R_{N G S}^{\mathrm{mod}}\left(C_{g N G S}^{\mathrm{mod}}-\hat{C}_{g N G S \text { nse }}^{\mathrm{mod}}\right)\left(R_{N G S}^{\mathrm{mod}}\right)^{T}\left(H_{T T}\right)^{T} .
$$

Note that the balanceG formula (2.4) defines a valid TT cova riance matrix provided the expression is SPD (wKich is expected if tKe simulation model is not tRo "far off" from the real system). Note also that for the ideal case of a simulation model perfectly matched to the system, (2.4) provides an exact estimate of the TT covariance matrix, which constitutes a strong result since the formula is therefore expected to yield reasonably accurate reconstructions for "small" simulation de-tunings (quantified in Section 3).

\section{PERFORMANCE EVALUATION}

In order to evaluate the performance of the TT PSFR algorithm defined in Section 2, a set of 50,000 stepsimulations were performed on a pair of GTX 580 Graphics Processing Units (GPUs) using the in-house developed Multi-threaded Adaptive Optics Simulator (MAOS) [18,19]. The TMT LGS MCAO system under design was simulated to high fiCelity, and the achieved simulation speed was $100 \mathrm{~ms} / \mathrm{step}$, which includes PSF aQd covariance matrix computations. TT PSFR Dcuracy has been assesseG by computing J band HQQFRed energy (EE) error cXrves,

$\left(E E_{T T}^{s y s}-\widehat{E E}_{T T}^{s y s}\right) / E E_{T T}^{s y s}$, whose values near the origin correspond to the TT SR error. $E E_{T T}$ is computed by integrating the product of a 2 dimensional sinc filter function with $O T F_{D L} K_{T T}$, where $O T F_{D L}$ denotes the diffraction limited OTF. These curves were computed on-axis. The 7-layer turbulence/wind profile shown in Table 1 has been simulated.

\begin{tabular}{|c|c|c|c|c|c|c|c|}
\hline Altitude (km) & 0 & 0.5 & 1 & 2 & 4 & 8 & 16 \\
\hline Wind Speed (m/s) & 5.6 & 5.8 & 6.2 & 7.6 & 13 & 19 & 12 \\
\hline Weights (\%) & 46 & 13 & 4 & 5 & 12 & 9 & 11 \\
\hline
\end{tabular}

Table 1 Turbulence profile relative weights and wind speed.

The profile was obtained from measurements performed during the 3-year TMT site testing cam paign on Mauna Kea [20]. The outer scale at each layer is $30 \mathrm{~m}$. Two profiles were simulated: a "baseline” profile yielding a Fried parameter of $18.6 \mathrm{~cm}$ (corLesponding to median Mauna Kea turbulence conditions), and a “09r0” profile yielding a Fri ed parameter of $16.7 \mathrm{~cm}$ (corresponding to roughly 65\% Mauna Kea turbulence cond itions). The isoplanatic angle is 2.2 arcsec, and the generalized isoplanatic angle for a deIormable mirror (DM) conjugation range of $11.2 \mathrm{~km}$ is 8.2 arc sec. The Greenwood frequency is $22 \mathrm{~Hz}$. All these atmospheric turbulence parameters are quoted at a reference wavelength of 500nm.

The system implements separate hi gh-order LGS and low-order NGS servo loops [21]. The L GS loop runs a tomographic minimum variance wavefront reconstruction algorithm at $800 \mathrm{~Hz}$ from measurements from 6 high-order LGS Shack-Hartmann WFSs (each of order 60 x 60). The NGS loop runs a classical least-squares reconstruction matrix, $R_{N G S}^{s y s}$, at low frame rate (generally around $90 \mathrm{~Hz}$ ), controlling $N_{m N G S}=5$ modes, defined as global TT and 3 TA modes distributed on the system's two DMs, from the combined measurements from a tip/tilt/focus/astigmatism (TTFA) NGS WFS (i.e. an order 2 x 2 Shack-Hartmann WFS) and two additional full-aperture TT NGS Shack-Hartmann WFSs [14]. The focus measurement from the TTFA NGS WFS is required in order to disentangle the atmospheric and mesospheric sodium layer focus errors. Regarding the servos, the LGS loop implements a simple type I servo with a gain of 50\%, whereas the NGS loop uses a more sophisticated type II servo, whose parameters (sampling frequency, modal gains and lead filter cross-over frequency and phase margin parameters) are obtained from an optimization procedure performed by a separate high-fidelity sky coverage simulation tool $[16,17]$. Sample NGS loop theoretical error rejection curves are plotted in Figure 1. Each curve is given by $10 \log _{10}\left(\left|1 /\left(1+H_{O L}\right)\right|^{2}\right)$, where $H_{O L}$ denotes the open-loop transfer function of the NGS loop (product of WFS, servo lag, digital-to-analog conversion, cascaded integrators and lead filter transfer functions [22]), and illustrates the charac teristic $-40 \mathrm{~dB}$ rejection per decade bel ow the $\sim 3 \mathrm{~Hz}-3 \mathrm{~dB}$ rejection bandwidth. 
The 4 different curves correspond to 4 different sets of servo parameters investigated in this paper (each optimized for a particular NGS asterism).

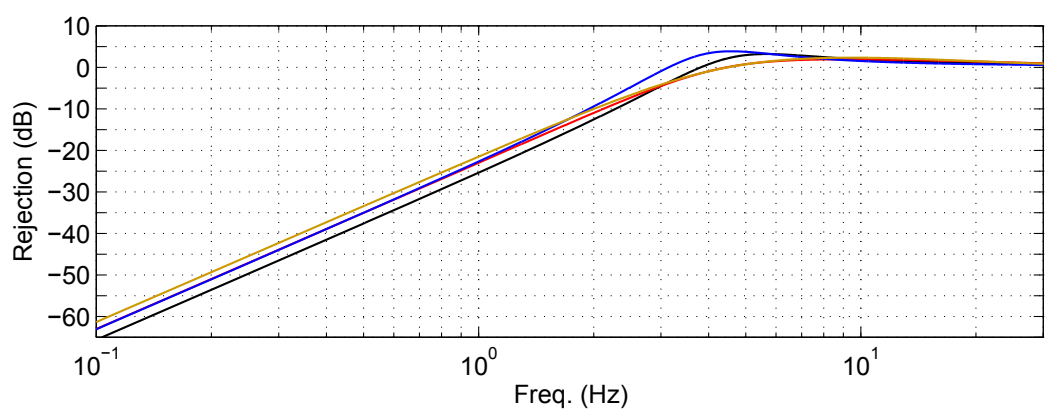

Figure 1: Sample error rejection curves for the NGS type II servo loop optimized for 4 sample NGS asterisms.

Finally, each NGS WFS subaperture is imaged by 4x4 detector pixels (Nyquist sampled in $\mathrm{H}$ band, i.e. 5.7mas pixel scale on the sky for the 2 TT WFSs and 11.4 mas pixel scale for the TTFA WFS), whose intensities are converted into average phase gradient by linear matched filter centroid weights. All these features are fully integrated in the simulation model. Readout noise was simulated at the level of 3 electrons RMS per read per pixel, both for the high-order LGS and low-order NGS WFSs.

We have performed the stu dy for 4 different NGS asterisms. Constellation geometries and star magnitudes are shown in the top panels of Figure 2. Each asterism consists of a TTFA and 2 TT stars. The black constellation (asterism 1) is an ideal $\backslash \backslash$ mmetrical asterism of stars of equal magnitude (19 in J band) on a 30"' radius circle. The red, blue and brown constellations (asterisms 2, 3,4) are asymmetrical (all within a 1' radius NGS patrol field) and of stars of different magnitude yielding around 60nm RMS residual NGS mode WFE (see Figure 3). Sampling frequencies are respectively 100,80, 90, and $66 \mathrm{~Hz}$ (resuCWof an optimization procedure performed by a sepaŁte high-fidelity sky coverage simulation tool [16,17]). Bottom panels display the signal-to-noise ratio (SNR) of each WFS (TTFA,TT,TT ordering), as well as the RMS noise equivalent angle in units of mas (NEA, i.e. the square root of the trace of $\hat{C}_{g N G S \text { nse }}^{s y s}$ at the sampling frequency of the loop. To give a point of comparison, for $D=30 \mathrm{~m}$, the diffraction limited J band PSF fullwidth at half maximum (FWHM) is $\lambda / D \sim 8.6 \mathrm{mas}$, and the TT WFSs pixel scale is 5.7mas (H band Nyquist sampling).
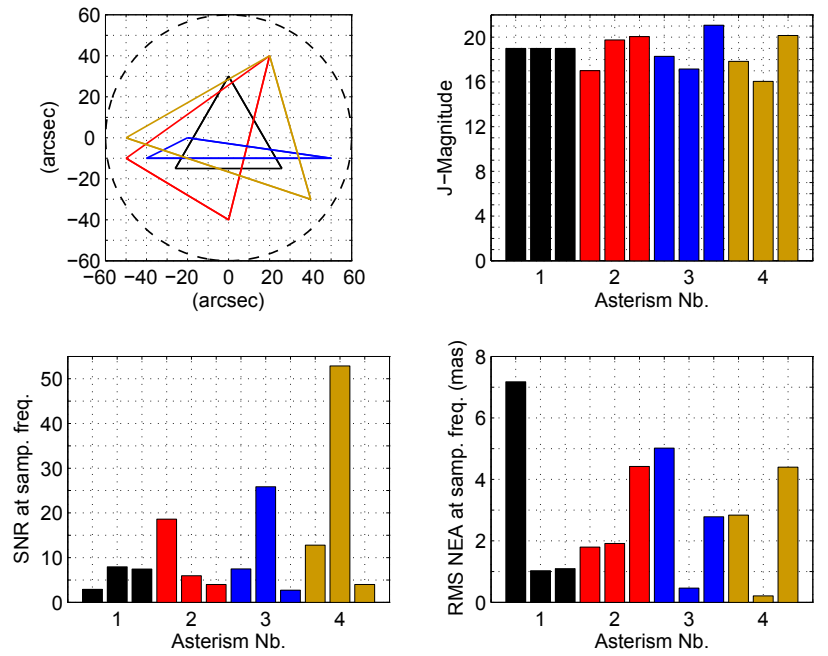

Figure 2: Top: geometry and star brightness of 4 sample NGS asterisms investigated in this paper. Bottom: signalto-noise ratio and RMS noise equivalent angle. 

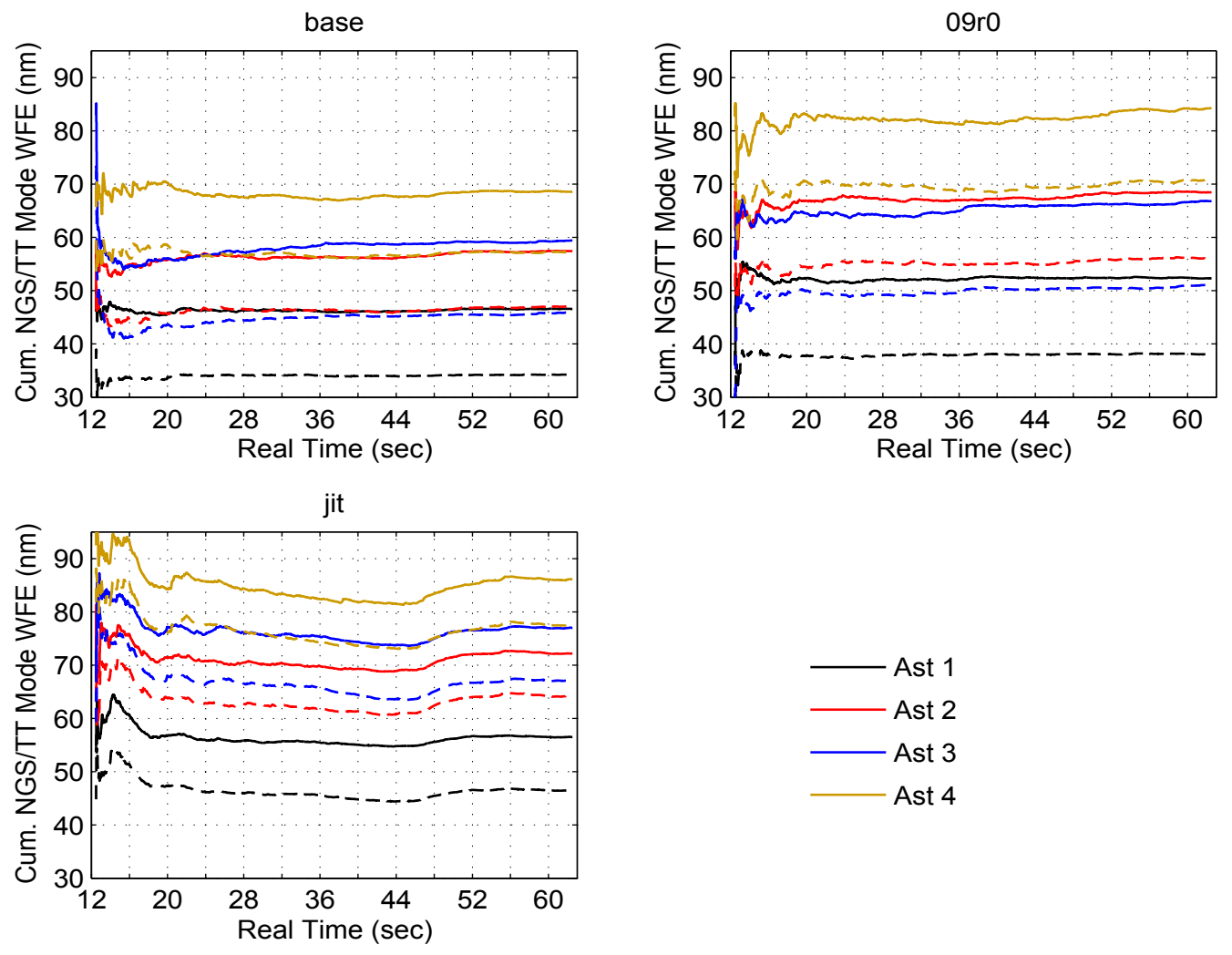

Figure 3: Cumulative NGS (solid) and TT (dashed) mode WFE averaged over a 17',x17', FoV for the baseline, 09r0, and jitter observing conditions. See text for details.

Figure 3 displays NGS loop performance during a $\sim 50$ s exposure time. Performance is given in term s of cumXative NGS modes (i.e. the 5 modes described in Section 2) and TT mode WFE averaged over a 17' 'x17', FoV. Performance has been averaged over 5 uncorrelated realizations of atmospheric frozen flow turbulence. The plots are indicators of NGS loop stability and stationarity. The iQtial transients observed in some of tKe curves disappear once the averaging time exceeds a few seconds. The cumXlative curves are otherwise very stable and flat, indicating that the NGS loop is stable and stationary for all cases investigated. The jitter case refers to the baseline condition with an additional injected TT jitter sine wave at $2 \mathrm{~Hz}$ and of RMS value equal to $\sigma_{\theta}=3$ mas angular displacement on the sky, i.e. a WFE of $\sigma_{T T}=D \sigma_{\theta} / 4 \sim 109 \mathrm{~nm}$. As seen from Figure 1, the NGS type II servo loop rejects this disturbance to $\sim-10 \mathrm{~dB}$.

Figure 4 illustrates sample TT PSFR accuracy results. Line colors follow Figure 3’s legend. Top panels are for ideal cases of a si mulation model perfectly matched to the syste m (including and excluding photon/read noise respectively). Such scenarios are clearly unrealistic, but of iP Sortance to assess tKe TT rec onstruction error for We unbalanced formulas (the balanced formulas yielding exact TT reconstructions in such cases). Salient features are:

1) The TT error (which is strongly NGS asterism dependent) is negative, i.e. the estim ated SR is too high (estimated TT covariance matrix is too weak)

2) The TT error is conce ntrated in the PSF core (in an area of size equal to roughl y twice the PSF FWHM), and gradually degrades between the different asterisms following Figure 3's NGS loop performance degradation. The Maréchal approximation relating SR loss to TT WFE $\sigma_{\theta} /(\lambda / D) \sim(2 / \pi) \sqrt{-\ln \left(S R_{T T}\right)}$, indicates that in ordeUto yield a SR estination error of respectiveQ $1 \%, 2 \%$ and $3 \%$, the R MS TT estim ation error, $\sigma_{\theta}$, has to be respectively equal to $6 \%, 9 \%$ and $11 \%$ of the diffraction limited PSF FWHM, $\lambda / D$, which places a c hallenging 
requirement on the TT PSFR algorithm. The fact that the unbalanced formula (2.2) does not generally do a good job at estimating the science TT covariance matrix suggests that it misses a tip/tilt anisoplanastism term or a high-order LGS-uncorrected atmospheric turbulence aliasing term or possibly a combination of both. This missing term is captured in the balanced formula (2.4). We should also point out that the simulation results discussed by Flicker in [1] are somewhat oversimplified since (i) the simulated atmosphere consisted of only TT and quadratic modes, and (ii) NGS WFSs were modeled using a geometrical linear model (infinite dynamic range).

3) The TT error is reduced in absence of photon and read-noise.
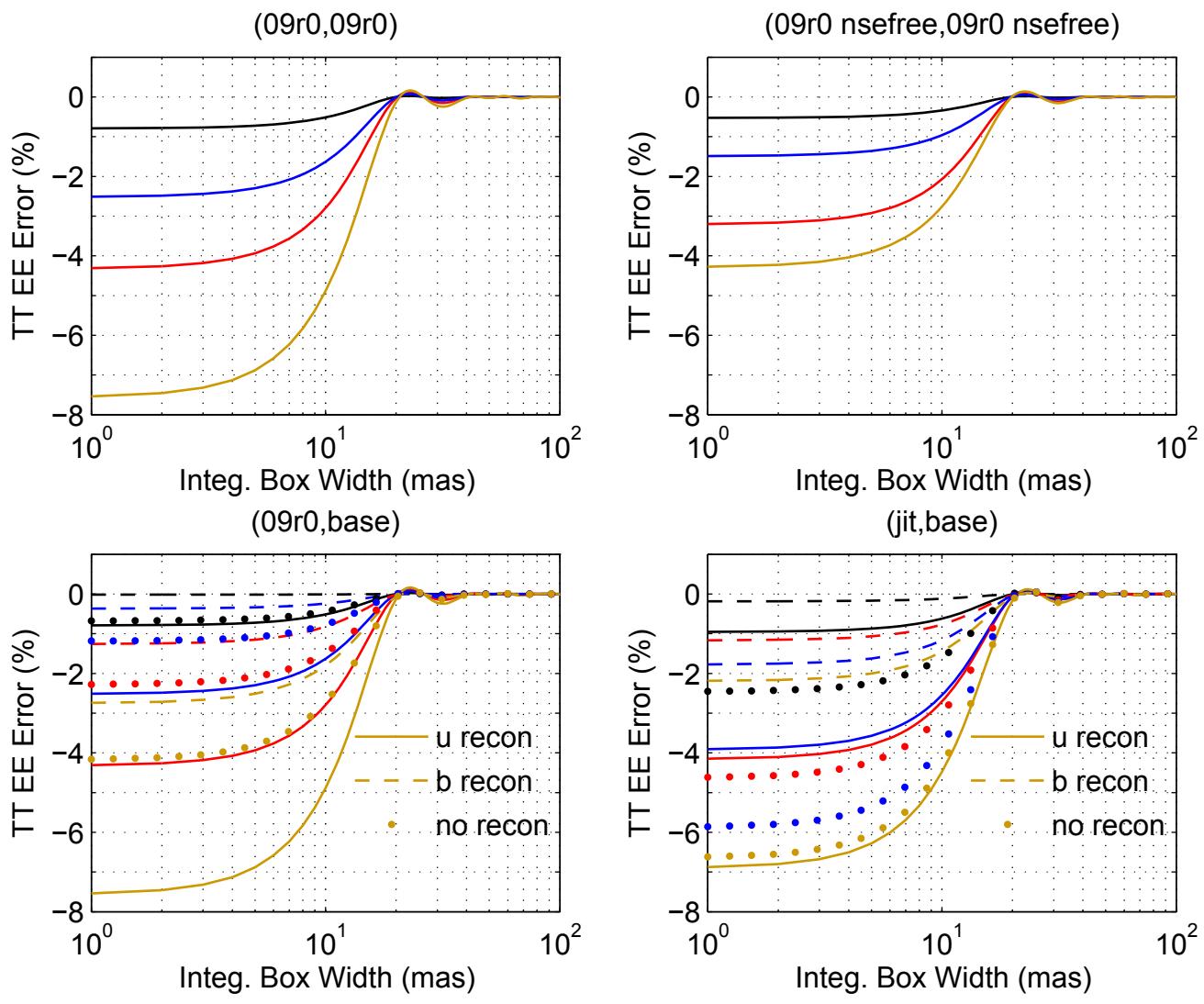

Figure 4: Top panels: on-axis TT EE reconstruction error for ideal cases of a simulation model perfectly tuned to the system (including and excluding photon/read noise respectively). Bottom panels: reconstruction error for de-tuned simulation models (by a 10\% Fried parameter error and an un-modeled 2Hz, 3mas TT jitter respectively). Solid lines are unbalanced errors, dashed lines are balanced errors and dotted lines a re errors made by relying exclusively on tKe simulation model (i.e. not performing any PSFR). See text for details.

The lower panels assess TT PSFR accuracy for the case of a simulation model detuned from the system by respectively a $10 \%$ Fried parameter error, and an un-modeled $2 \mathrm{~Hz}$, 3mas TT jitter. Salient features for the case of a simulation model detuned in seeing are:

1) The unbalanced TT error shows the same pathology that was discussed in the upper left panel since the unbalanced formula is model independent and the system is in the same observing condition as it was in the upper right panel. 
2) The balanced TT error is signi ficantly reduced compared to the unbalDhced error: 3 out of the 4 NGS asterisms investigated yield sub-percent TT SR errors.

Salient features for the case of an un-modeled TT jitter are:

1) The balanced TT error is si gnificantly smaller than the unbalanced error, but a resiGual SR error remains, whose magnitude is at or above the percent level.

\section{CONCLUSIONS}

We have discussed the problem of TT PSFR for / *6 MCAO. The approach is bas ed on the schemHdeveloped by Flicker in 2003 [1], and consists in post-processing the measurement covariance matrix of multiple low-order NGS WFSs. An innovative simulation model based "balanced" algorithm has been iQtoduced to caSture error terms not accounted for in Flicker's algorithm. These results demRnstrate the superiority of the balanced method and call for further analytical work and experimental validation.

\section{ACKNOWLEDGEMENTS}

The TMT Project gratefully acknowledg es the support of tKe TMT collaborating institu tions. They are tKe Association of Canadian Universities for Research in Astronomy (ACURA), the California Institute of Technology, the University of California, the National Astronom ical Observatory of Japan, the National Astronomical Observatories of China and their consortium partners, and the DeSartment of Science a nd Technology of In dia and their suSported institutes. This wolk was supported as well by the Gordon and Betty Moore FoundDibn, the Canada Foundation for Innovation, the Ontario Ministry of Research and Innovation, the National Research Council of Canada, the Natural Sciences and Engineering Research Council of Canada, the British Columbia Knowledge Development Fund, the Association of Universities foUResearch in Astronom\ (AURA) and the U. S. National Science FoXndation. L.Gilles's email address is lgilles@caltech.edu.

\section{REFERENCES}

1. R.Flicker, F.Rigaut and B.Ellerbroek, “'Tilt anisoplanatism in laser guide star based multi conjugate adaptive optics. Reconstruction of the long exposure point spread function from control loop data”, Astron. Astrophys. 400, 11991207 (2003).

2. P.B.Cameron, M.C.Britton and S.R.Kulkarni, "Precision astrometry with adaptive optics," in "Adaptive Optics Systems," N.N.Hubin, C.E.Max and P.L.Wizinowich, eds., Proc. Soc. Photo-Opt. Instrum. Eng. 7015, 70150A-1 70150A-9 (2008).

3. P.B.Cameron, M.C.Britton and S.R.Kulkarni, "Precision astrometry with ada ptive optics,” Astron. J. 137, 83-93 (2009).

4. C.Schödel, "Accurate phot ometry with adaptive optics in the pr esence of anisoplaQatic effects with spađely sampled PSF: the Galactic center as an e[ ample of a challenging target for accura te AO phot ometry," Astron. Astrophys. 509, A58 (2010).

5. R.Davies, H.Engel, E.Hicks, N.F.Schreiber, R.Genzel, L.Tacconi, F.Ei senhauer, S.Rabien, “Dissecting Galaxies with Adaptive Optics," in “Adaptive Optics Systems II," B.L.Ellerbroek, M.Hart, N.Hubin, P.L.wizinowich, eds., Proc. Soc. Photo-Opt. Instrum. Eng. 7736, 7736G-1 - 7736G-9 (2010).

6. M.Ammons, E.Bendek and O.Guyon, "Microarcsecond relative astrometry from the grRund with a diffractive pupil,” Proc. Soc. Photo-Opt. Instrum. Eng. 8151, 81510T (2011). 
7. G.Herriot, D.Andersen, J.Atwood, C.Boyer, A.Beauvillier, P.Byrnes, R.Conan, B.Ellerbroek, J.Fitzsimmons, L.Gilles, P.Hickson, A.Hill, K.Jackson, O.lardière, J.Pazder, T.Pfrommer, V.Reshet ov, S.Roberts, J.-P.Véran, L.Wang and I.Wevers, "NFIRAOS: TMT's facility adap tive optics system," in "Adaptive Optics II," B.L.Ellerbroek, M.Hart, N.Hubin and P.L.Wizinowich, eds., Proc. Soc. Photo-Opt. Instrum. Eng. 7736, 77360B-1 77360B-8 (2010).

8. B.Ellerbroek, S.Adkins, D.Andersen, J.Atwood, S.Browne, C.Boyer, P.Byrnes, K.Caputa, R.Conan, R.Cousty, D.Erikson, J.Fitzsimmons, F.Gamache, L.Gilles, G.Herriot, P.Hickson, O.Lardiere, P.Morin, J.Pazder, T.Pfrommer, D.Quinn, V.Reshetov, S.roberts, J.-C.Sinquin, M.Schoeck, M.Smith, G.Tyler, J.Vaughn, J.-P.Véran, C.vogel, L.Wang and I.Wevers, "First light a daptive optics system s and components for the Thirty Meter Telescope", in "Adaptive Optics Systems II," B.L.Ellerbroek, M.Hart, N.Hubin and P.L.Wizinowich, eds., Proc. Soc. Photo-Opt. Instrum. Eng. 7736, 773604-1 - 773604-14 (2010).

9. J.P.Véran F.Rigaut, H.Maitre and D.Rouan, “Estimation of the adaptive optics long-exposure point-spread function using control loop data," J. Opt. Soc. Am. A 14, 3057-3069 (1997).

10. T.Fusco, J.-M.Conan, L.M.Mugnier, V.Michau and G.Rousset, "'Characterization of adaptive optics point spread function for anisoplanatic imaging. Application to stellar field deconvolution," Astron. Astrophys. Suppl. Ser. 142, 149-156 (2000).

11. M.C.Britton,"'The anisoplanatic point-spread function in adaptive optics," Pub. Astron. Soc. Pac. 118, 885-900 (2006).

12. L.Jolissaint, C.Neyman, J.Christou, P.Wizinowich and L.Mugnier, "First successful adaptive optics PSF reconstruction at W.M.Keck observatory,” http://arxiv.org/abs/1202.3486 (2012).

13. R.Flicker,"PSF reconstruction for Keck AO," W.M.Keck Observatory, 65-1120 Mamalahoa Hwy., Kamuela, HI 96743, USA (unpublished).

14. B.Ellerbroek and F.Rigaut, "Methods for correcting tilt anisoplanatism in laser guide star based multiconj ugate adaptive optics,” J. Opt. Soc. Am. A 18, 2539-2547 (2001).

15. L.Gilles, C.Correia, J.P.Véran, L.Wang and B.Ellerbroek, "A simulation model based approach for long exposure atmospheric point spread function reco nstruction for laser guide star multi conjugate adaptive optics," submitted to Appl. Opt. (2012).

16. L.Wang, B.Ellerbroek and J.P.Véran, "High fidelity sky coverage analysis via time dom ain adaptive optics simulations,” Appl. Opt. 48, 5076-5087 (2009).

17. L.Wang, B.Ellerbroek and J.P.Véran, "Full physical optics sky coverage simulation for MCAO systems on ELTs,” 1st Adaptive Optics for Ex tremely Large Telescopes conference (2010). Available online a t http://dx.doi.org/10.1051/a04elt/201003003

18. L.Wang and B.L.Ellerbroek, "Fast end-to-end multi-conjugate AO simulation using graphical processing units and the maos simulation code”, Proc. “Adaptive Optics for Extremely Large Telescopes II” (2012).

19. http://lianqiw.github.com/maos

20. M.Schoeck, S.Els, R.Riddle, W.Skidmore, T.Travouillon, R.Blum, E.Bustos, G.Chanan, S.G.Djorgovski, P.Gillett, B.Gregory, J.Nelson, A.Otarola, J.Seguel, J.Vasquez, A.Walker, D.Walker and L.Wang, "Thirty Meter Telescope Site Testing I: Overview,” Pub. Astron. Soc. Pac. 121, 384-395 (2009).

21. L.Gilles and B.Ellerbroek, "Split atmospheric tomography using laser and natural guide stars," J. Opt. Soc. Am. A 25, 2427-2435 (2008). 
22. J.P.Véran and G.Herriot, "Type II w oofer-tweeter control for NFIRAOS on TMT,” in "Adaptive optics: methods, analysis and applications,” Optical Society of America technical digest (CD), paper JTuC2 (2009).

Proc. of SPIE Vol. $8447844729-9$

Downloaded From: http://proceedings.spiedigitallibrary.org/ on 01/24/2013 Terms of Use: http://spiedl.org/terms 